\title{
Meteoric dust effects on D-region incoherent scatter radar spectra
}

\author{
John Y. N. Cho, ${ }^{* 1}$ Michael P. Sulzer' and Michael C. Kelley ${ }^{2}$ \\ ${ }^{1}$ A recibo Observatory, P.O. Box 995, Arecibo, Puerto Rico 00613, U.S.A.; ${ }^{2}$ School of Electrical Fngineering, \\ Cornell University, Ithaca, New York 14853, U.S.A.
}

Reccited 25 June 1997 ; in revised form 8 July 1997 ; accepted 15 August 1997

\begin{abstract}
We extend the incoherent backscatter radar equation for the weakly ionized, collision-dominated case to include polydisperse charged dust, using the fluid-equation approach. Calculations with this equation show that the main effect of charged dust on the radar Doppler spectrum is to narrow it, except in the case of very small, negatively charged dust, which widens the spectrum. The results are encouraging for the use of incoherent scatter radar as an instrument for meteoric dust measurement. We also note that if the effects of meteoric dust are not taken into account, one may get a systematic bias in the D-region parameters deduced from the radar spectra. This may account for the discrepancy between observed and calculated spectra reported in earlier papers. 1998 Elsevier Science Ltd. All rights reserved
\end{abstract}

\section{INTRODUCTION}

Watching meteors at night is a favorite pastime of people all over the world. Research in past decades has shown that most of the meteoric ablation that result in bright visual streaks and strong radar returns occur in the $80-100 \mathrm{~km}$ height region. However, despite the meteor's familiarity and long history as an object of scientific study, little is known about what happens to the estimated $100+$ metric tons per day (Love and Brownlee, 1993) that enters our atmosphere. It is widely assumed that the vaporized material recondense and form smoke particles (Rosinski and Snow, 1961), which then coagulate and sediment downward through the atmosphere, but there have been no confirmed measurements of such aerosols in the ablation zone. The most promising report so far has been the detection of negatively charged microclusters by a rocket-borne mass spectrometer (Schulte and Arnold, 1992).

Dust plays a crucial role in the atmosphere by acting as nucleation sites for cloud growth. We expect meteoric dust to be especially important in the stratosphere and mesosphere, where tropospheric dust has trouble reaching except in cases of major volcanic eruptions. Meteoric dust is one of the most likely nucleation sites for noctilucent clouds (polar mesospheric clouds) (Thomas, 1991), and they may also

\footnotetext{
* Now at the Department of Earth, Atmospheric, and Planetary Sciences, Massachusetts Institute of Technology, Koom 54-1823, Cambridge, Massachusetts 02139, U.S.A.
}

serve as condensation nuclei for stratospheric $\mathrm{H}_{2} \mathrm{SO}_{4} / \mathrm{H}_{2} \mathrm{O}$ droplets and polar stratospheric clouds (Turco et al., 1981) that play a critical rolc in the destruction of the ozone layer (Solomon, 1990).

Radars have long been used to observe meteors for their intrinsic study as well as for their use as wind tracers. Unfortunately, detection of meteoric dust by radar has, so far, not been possible. However, by a fortuitous coincidence, a rocket launched to study other phenomena appeared to have flown right through a meteor trail that was simultaneously observed by a coherent-scatter radar. This serendipity allowed us to estimate some meteoric dust parameters (see companion paper by Kelley et al. (1997)). In the future we would like to be able to measure meteoric dust parameters without having to rely on such improbable events. Thus, in this paper we lay the ground work for a possible systematic observation of meteoric dust in the ablation zone using incoherent-scatter radars (ISRs).

\section{DERIVATION OF THE BACKSCATTER SPECTRAI EQUATION}

The meteoric ablation zone lies in the D-region where the incoherent (Thomson) backscatter of radar waves yields Doppler spectra with shapes that are determined by the state of the weakly ionized plasma and neutral gas mixture. Although the radiowaves are scattered by thermally induced Bragg-scale structures in the free electron density, the behavior of the electrons are strongly coupled to the ions through ambi- 
polar electric fields. And because the mean free paths of the ions are much shorter than the ISR Bragg length, the spread in the Doppler spectrum of what is called the ion line is proportional to the ion diffusivity. In this way some parameters of the ions can be deduced.

Because meteoric dust likely spans a range of sizes, we need an ISR theory that can accommodate polydisperse charged aerosols. In this section we develop a radar backscatter equation that can handle any number of charged species. We will closely follow the continuum approach used by Tanenbaum (1968) and Mathews (1978). Since there have been many papers in the past that have detailed the various formalisms for incoherent scatter calculations, we will restrict ourselves to describing the extension of the three-fluid rcsult of Mathews (1978) to the arbitrary $N$-fluid case.

The backscatter cross section for ISR is

$$
\sigma_{b}\left(\omega_{0}+\omega\right) \mathrm{d} \omega=V r_{e}^{2}\left\langle\left|\Delta N_{e}(k, \omega)\right|^{2}\right\rangle \mathrm{d} \omega
$$

where $\omega_{0}$ is the radar frequency, $\omega$ is the Doppler frequency shift from $\omega_{0}, V$ is the radar volume, $r_{e}$ is the classical electron radius, $\Delta N_{e}$ is the electron density fluctuation spectrum, and $k$ is the backscatter radar Bragg wavenumber. The continuity equation

$$
\frac{\partial \Delta N_{e}}{\partial t}+\nabla \cdot \Gamma_{e}=0
$$

where $\Gamma_{e}$ is the electron flux density along the radar beam yields, with Fourier decomposition,

$$
\left\langle\left|\Delta N_{e}\right|^{2}\right\rangle=\frac{k^{2}}{\omega^{2}}\left\langle\left|\boldsymbol{\Gamma}_{\ell}\right|^{2}\right\rangle .
$$

Thus, we have

$$
\sigma_{b}\left(\omega_{0}+\omega\right) \mathrm{d} \omega=\frac{V r_{e}^{2} k^{2}}{\omega^{2}}\left\langle\left|\Gamma_{e}\right|^{2}\right\rangle \mathrm{d} \omega
$$

by substituting eqn (3) into eqn (1).

The flux density of a given constituent, $\Gamma_{s}$, can be related to an applied force, $F_{s}$, through the complex admittance function, $Y_{s}$, to give

$$
\Gamma_{s}=Y_{s}\left(F_{s}+Z_{s} e E\right)
$$

where $Z_{s}$ is the charge number of the species, $e$ is the electron charge, and $E$ is the mutual electric field of the plasma. Note that forces due to the magnetic field are ignored and the vector notation is dropped, since the high collision frequency wipes out magnetic effects.

From Maxwell's equation we have, for $\mathbf{V} \times \mathbf{H}=\mathbf{0}$,

$$
\frac{\partial \mathbf{D}}{\partial t}+\mathbf{J}=\mathbf{0}
$$

where $\mathbf{D}$ is the displacement vector and $\mathbf{J}$ is the current density. Again, with Fourier decomposition, we get

$$
-Y_{\mathrm{I}} \mathrm{e}^{2} E=J=e \sum_{s} Z_{s} \Gamma_{s}
$$

where $Y_{1} \equiv i \omega \varepsilon_{0} / e^{2}$ and $\varepsilon_{0}$ is the free space permittivity. We see that eqn (5) and eqn (7) give us $N+1$ equations for $N+1$ unknowns given $N$ species, so after some algebra we are able to solve for the electron flux density

$$
\Gamma_{e}=\frac{Y_{\mathrm{I}} \mid \sum_{s \neq e} Z_{s} Y_{s}\left(Z_{s} F_{e}+F_{s}\right)}{Y_{\mathrm{I}}+Y_{e}+\sum_{s \neq e} Z_{s}^{2} Y_{s}} Y_{e}
$$

where the summations have now excluded the electron terms. Taking the ensemble average of eqn (8) we get

$$
\begin{aligned}
& \left\langle\left|\Gamma_{c}\right|^{2}\right\rangle=\left|\frac{Y_{e}}{Y_{\mathrm{I}}+Y_{e}+\sum_{s \neq e} Z_{s}^{2} Y_{s}}\right|^{2} \\
& \times\left(\left|Y_{\mathrm{I}}+\sum_{s \neq e} Z_{s}^{2} Y_{s}\right|^{2}\left\langle\left|F_{e}\right|^{2}\right\rangle+\sum_{v \neq e} Z_{s}^{2}\left|Y_{s}\right|^{2}\left\langle\left|F_{s}\right|^{2}\right\rangle\right) .
\end{aligned}
$$

We now make use of the Nyquist fluctuation dissipation theorem to relate the forcing functions to the admittance functions (Dougherty and Farlcy, 1960)

$$
\left\langle\left|F_{\mathrm{s}}\right|^{2}\right\rangle \mathrm{d} \omega=\frac{k_{\mathrm{B}} T_{s}}{\pi V^{V}} \operatorname{Re}\left(Y_{s}^{-1}\right) \mathrm{d} \omega
$$

where $k_{\mathrm{B}}$ is the Boltzmann constant and $T_{s}$ is the temperature of the species. Combining eqn (9) and eqn (10) we get

$$
\begin{aligned}
\left\langle\left|\Gamma_{e}\right|^{2}\right\rangle \mathrm{d} \omega & =\frac{k_{\mathrm{B}}}{\pi V}\left|\frac{Y_{e}}{Y_{\mathrm{I}}+Y_{e}+\sum_{s \neq e} Y_{s}}\right|^{2} \\
& \times \operatorname{Re}\left(\left|Y_{\mathrm{I}}+\sum_{s \neq e} Y_{s}\right|^{2} \frac{T_{e}}{Y_{e}}+\sum_{s=e}\left|Y_{s}\right|^{2} \frac{T_{s}}{Y_{s}}\right) .
\end{aligned}
$$

Note that we have let $Z_{s}^{2}=1$ at this point. Multiple units of charge per particle causes a Debye sphere of electron surplus/debit to form around the high- $Z$ particle, resulting in enhanced scattering due to those electrons responding in phase to the incident wave. The equations we are using here do not encompass this phenomenon, so we refer the reader interested in the multiple-charge case to other papers (Hagfors, 1992; LaHoz, 1992; Trakhtengerts and Demekhov, 1995). In any case, singly charged particles is a good 
assumption up to about $10-\mathrm{nm}$ radius aerosols if photoelectric charging is negligible (Jensen and Thomas, 1991).

We now need to find a physical expression for the admittance function of each species. Here we simply appropriate the results from the fluid-equation approach used by Tanenbaum (1968), where linearized mass, momentum, and energy transport equations for each constituent along with the ideal gas law are combined to yield

$$
Y_{s}=Y_{\mathrm{I}} \frac{\alpha_{s}^{2}}{z_{s}}
$$

where

$$
\alpha_{s}=\frac{1}{k \lambda_{\mathrm{D} s}}=\frac{e}{k}\left(\frac{N_{s}}{\varepsilon_{0} k_{\mathrm{B}} T_{s}}\right)^{1 / 2}
$$

and $\lambda_{\mathrm{D} s}$ is the Debye length, and $N_{s}$ is the number density of the sth component. Furthermore,

$$
z_{s}=\frac{1+i \frac{5 \theta_{s}}{3 \sigma_{s}}}{1+i \frac{\theta_{s}}{\sigma_{s}}}+2 i \theta_{s}\left(\psi_{s}+\frac{2}{3 d_{s} \psi_{s}}\right)-2 \theta_{s}^{2}
$$

where $\theta_{s}=\omega / 2^{1 / 2} k v_{s}$ is the normalized frequency, $v_{s}=\left(k_{\mathrm{B}} T_{s} / m_{s}\right)^{1 / 2}$ is the mean thermal velocity, $m_{s}$ is the component mass, $\psi_{s}=v_{s n} / 2^{1 / 2} k v_{s}$ is the normalized constituent-neutral collision frequency, and

$$
\sigma_{s}=\frac{2 m_{s} \psi_{s}}{m_{s}+m_{n}}+\frac{5}{4 c_{s} \psi_{s}} .
$$

For the viscosity and thermal conductivity constants we choose $d_{s}=1.55$ for $s=e, d_{s}=1.78$ for $s \neq e$, $c_{s}=1.5$ for $s=e$, and $c_{s}=2.28$ for $s \neq e$ (Tanenbaum, 1968). These values are appropriate for Maxwellian interparticle forces. If the constituent is larger than a threshold radius (to be discussed below), then we switch to hard-sphere interaction values of $d_{s}=1.6$ and $c_{s}=2.1$.

The electron-neutral collision frequency is (Banks and Kockarts, 1973)

$$
v_{e n}=\left(3.78 \times 10^{-11} T_{e}^{1 / 2}+1.98 \times 10^{-11} T_{e}\right) N_{n}
$$

where the neutral number density, $N_{n}$, is specified in units of $\mathrm{cm}^{-3}$. For the non-electron constituents, Cho et al. (1992) showed that the collision frequency with the neutrals passes from a regime of polarization interaction to that of hard-sphere interaction when the particle radius becomes larger than a certain radius, which is of the order of $0.5 \mathrm{~nm}$ in the upper mesosphere. In computing the spectrum one can calculate both the polarization and the hard-sphere cases and use the higher value for a given particle radius. The polarization collision frequency is (Banks and Kockarts, 1973)

$$
v_{s n}^{P}=2.59 \times 10^{-9} \frac{N_{n}}{M_{s}^{1 / 2}} \sum_{t} F_{\imath}\left(\frac{M_{n i} \chi_{n t}}{M_{s}+M_{n t}}\right)^{1 / 2}
$$

where $N_{n}$ is specified here in units of $\mathrm{cm}^{-3}, M_{s}$ is the mass of the charged species in atomic mass units (amu), the summation of $t$ is taken over the neutral constituents, $F_{t}$ is the fractional volume of each neutral gas, $M_{n t}$ is the mass of the neutral component in amu, and $\chi_{n t}$ is the polarizability of the neutral gas in units of $10^{-24} \mathrm{~cm}^{-3}$. The atmospheric mixture is $78 \%$ $\mathrm{N}_{2}, 21 \% \mathrm{O}_{2}$, and $1 \% \mathrm{Ar}$, with $\chi_{n t}$ of $1.74,1.57$, and 1.64 , respectively. The hard-sphere collision frequency is (Schunk, 1975)

$$
v_{s n}^{H}=\frac{8\left(r_{s}+r_{n}\right)^{2} N_{n}}{3\left(m_{s}+m_{n}\right)}\left[\frac{2 \pi k_{\mathrm{B}} m_{n}\left(m_{s} T_{n}+m_{n} T_{s}\right)}{m_{s}}\right]^{1 / 2}
$$

where $r_{s}$ is the constituent radius, $r_{n}=0.15 \mathrm{~nm}$ is the mean neutral radius, $m_{n}=3.85 \times 10^{-26} \mathrm{~kg}$ is the mean neutral mass, and $T_{n}$ is the neutral temperature. Note that all the quantities in eqn (18) are specified in standard MKS units.

Finally we can substitute eqn (12) into eqn (11), then plug the result into eqn (4) to arrive at the final result

$$
\begin{aligned}
\sigma_{b}\left(\omega_{0}+\omega\right) \mathrm{d} \omega & =\frac{r_{e}^{2} N_{e} \mathrm{~d} \omega}{\pi \omega}\left|\frac{1}{\alpha_{e}^{2}+z_{e}\left(1+\sum_{s \neq e} \frac{\alpha_{s}^{2}}{z_{s}}\right)}\right|^{2} \\
& \times \operatorname{Im}\left(\left|1+\sum_{s \neq e} \frac{\alpha_{s}^{2}}{z_{s}}\right|^{2} z_{e}+\frac{\alpha_{e}^{2}}{T_{e}} \sum_{s \neq e} T_{s} \frac{\alpha_{s}^{2}}{z_{s}^{*}}\right)
\end{aligned}
$$

where the asterisk denotes the complex conjugate. At $\omega=0$, eqn (19) becomes

$$
\sigma_{b}\left(\omega_{0}\right) \mathrm{d} \omega=\frac{r_{e}^{2} N_{e} \mathrm{~d} \omega}{2^{1 / 2} \pi k} \frac{\left|1+\sum_{s \neq e} \alpha_{s}^{2}\right|^{2} \frac{\beta_{e}}{v_{e}}+\frac{\alpha_{e}^{2}}{T_{e}} \sum_{s \neq e} T_{s} \alpha_{s}^{2} \frac{\beta_{s}}{v_{s}}}{\left|\alpha_{e}^{2}+1+\sum_{s \neq e} \alpha_{s}^{2}\right|^{2}}
$$

where $\beta_{s}=2 / 3 \sigma_{s}+2\left[\psi_{s}+2 /\left(3 d_{s} \psi_{s}\right)\right]$. 


\section{SPECTRAL CalCUlations}

We now have the proper tool for exploring the effects of polydisperse charged dust on D-region ISR spectra. In the following computations we will fix the values of certain parameters to constants that are typical around a height of $90 \mathrm{~km}: N_{e}=5000 \mathrm{~cm}^{-3}$, $N_{n}=1.5 \times 10^{14} \mathrm{~cm}^{-3}$, and $T_{s}=T_{n}=200 \mathrm{~K}$. Furthermore, we will fix the radar frequency to be $430 \mathrm{MHz}$ and assume a mass density of $2 \mathrm{~g} \mathrm{~cm}^{-3}$ for the dust material following Hunten et al. (1980). The constituents will be only singly charged. The usual normalizations of $\pi^{1 / 2} N_{e}^{-1} r_{e}^{-2} \sigma_{b}$ and $\theta_{i}$ will be used for the ordinate and abscissa, where a 31-amu ion is used to define $\theta_{i}$. Only the "ion line" portion of the spectra will be plotted, since the electron line is not affected by the changes in the dust-size distribution.

In Fig. 1, the thick line represents the case where a 31 -amu positive ion is the only non-electron component ; this curve will be reproduced in all subsequent spectral plots as a reference. The dashed curve is the calculation for two positive components : $2500 \mathrm{~cm}^{-3}$ of $5-\mathrm{nm}$ radius particles in addition to $2500 \mathrm{~cm}^{-3}$ of the 31-amu ions. Note the introduction of a narrower spectral line corresponding to the larger species. The

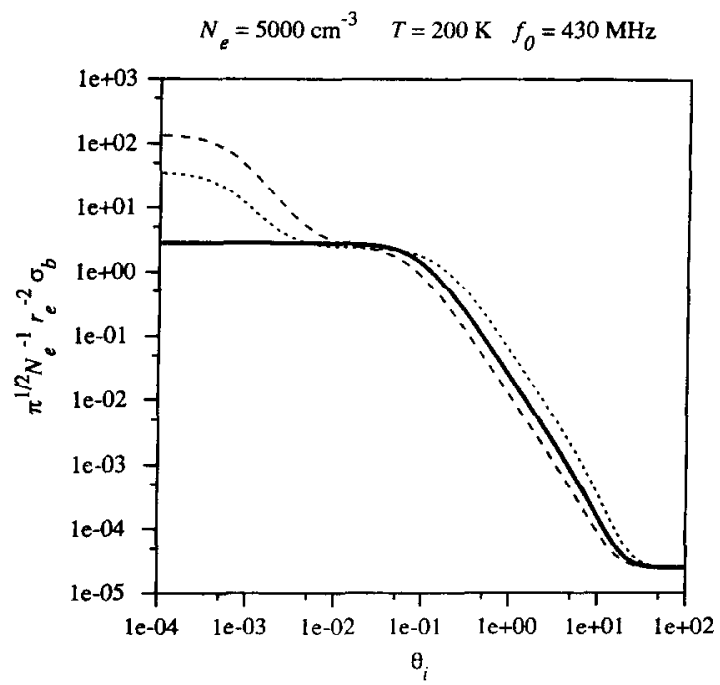

Fig. 1. The dashed curve is the calculated incoherent scatter spectra for a plasma with $2500 \mathrm{~cm}^{-3} 31$-amu positive ions and $2500 \mathrm{~cm}^{-3}$ positively charged $5-\mathrm{nm}$ radius dust particles. The dotted curve is for a plasma with $2500 \mathrm{~cm}^{-3} 31$-amu negative ions, $2500 \mathrm{~cm}^{-3}$ negatively charged $5-\mathrm{nm}$ particles, and $10,000 \mathrm{~cm}^{-3} 31$-amu positive ions. The thick line is the reference spectrum with only $5000 \mathrm{~cm}^{-3} 31$ amu positive ions to neutralize the electrons. The neutral density used throughout is $1.5 \times 10^{14} \mathrm{~cm}^{-3}$. The normalization of the axes is explained in the text. dotted curve is for the addition of two more negative species to the reference mixture: $2500 \mathrm{~cm}^{-3}$ of 31 amu negative ions and $2500 \mathrm{~cm}^{-3}$ of $5-\mathrm{nm}$ negatively charged dust; the number of reference positive ions has been increased to $10,000 \mathrm{~cm}^{-3}$ to keep the plasma neutral. Note that there is both a widening of the original "ion line" and the appearance of a narrower line. The former is due to the light negative ions (an effect already noted by Mathews (1978)) and the latter is caused by the large dust particles. This transition of the negative species from a special widening to a narrowing agent occurs when the particle radius is around $1 \mathrm{~nm}$ (Cho et al., 1996). For positive species the effect is always a narrowing of the spectrum with increasing size.

We now attempt to compute some spectra for more rcalistic dust size distributions. We look to the model calculations of Hunten et al. (1980) (henceforth referred to as HTT80) for guidance. The greatest variable in their model is the initial smoke particle size, which can weight the dust size distribution to a large number of small particles or a small number of large particles. Since number densities much smaller than our reference electron density of $5000 \mathrm{~cm}^{-3}$ would not influence the ISR spectrum very much, we stick to the HTT80 dust distributions with small initial smoke sizes.

Figure 2 shows the spectra calculated for two size distributions given in Table 1 . The dotted curve cor-

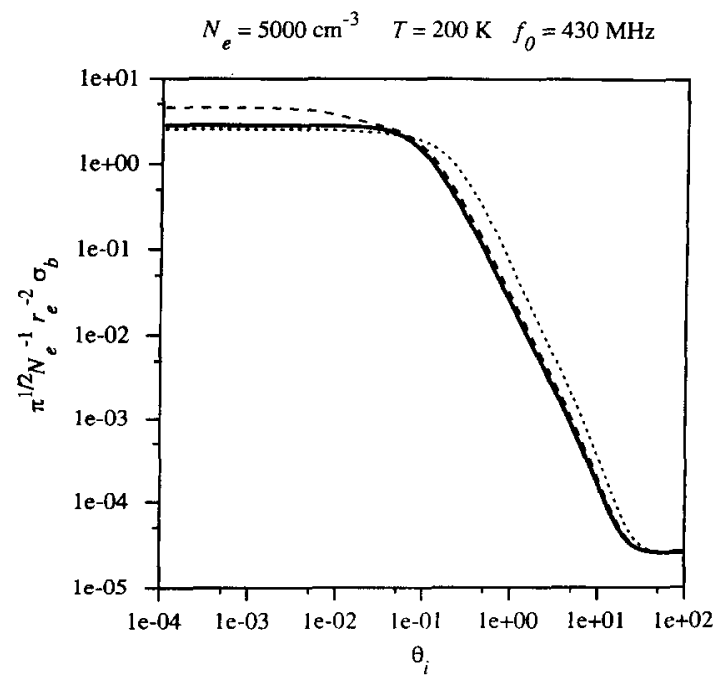

Fig. 2. Calculated incoherent scatter spectra for parameters given in Table 1. (a) is for the dotted curve and (b) is for the dashed curve. The thick line is the reference spectrum for Fig. 1. 
Table 1. Charge and size distributions of the non-electron components used to produce the spectra in Fig. 2. (a) is for the dotted curve and (b) is for the dashed curve

\begin{tabular}{rrrl}
\hline & $N_{s}\left(\mathrm{~cm}^{-3}\right)$ & $Z_{s}$ & $r_{s}(\mathrm{~nm})$ \\
\hline 11,870 & 1 & 0.18 \\
5000 & -1 & 0.5 \\
1000 & -1 & 0.6 \\
500 & -1 & 0.7 \\
200 & -1 & 0.8 \\
100 & -1 & 0.9 \\
70 & -1 & 1 \\
& 6130 & 1 & 0.18 \\
& 1000 & -1 & 1 \\
100 & -1 & 1.2 \\
16 & -1 & 1.4 \\
8 & -1 & 1.6 \\
4 & -1 & 1.8 \\
& 2 & -1 & 2 \\
\hline
\end{tabular}

responds to the smaller size distribution, which roughly follows the HTT80 model with an initial smoke particle radius of $0.5 \mathrm{~nm}$. The dashed curve is for a larger size distribution with an initial smoke radius of $1 \mathrm{~nm}$. In both cases the dust particles are only negatively charged (a sound assumption if photoelectric charging is negligible). The population of reference positive ions is increased to maintain charge balance. As might be expected the smaller distribution of dust widens the spectrum, whereas the larger distribution narrows it.

Table 2 gives the parameters used in Fig. 3. The dotted curve corresponds to an all-positive dust distribution with an initial smoke particle radius of $1 \mathrm{~nm}$. As expected the result is a spectral narrowing. The dashed curve is for the case where an equal number of dust particles are charged both negatively and positively at each size. Such a mixture of negatively and positively charged dust may occur if photoelectric charging is effective for a portion of the dust population. There is apparently some tendency for the plus and minus components to counterbalance each other, but the overall effect is still mainly a spectral narrowing.

As one can see from these plots the difference between a spectrum with dust and one without can be fairly subtle for what we believe to be a realistic dustsize distribution (e.g., Fig. 2). Therefore, we need to investigate whether systematic, dust-caused spectral distortions can be distinguished from statistical deviations away from the standard, dust-free model spectrum.

To simulate the fitting of real data, we take the
Table 2. Charge and size distributions of the non-electron components used to produce the spectra in Fig. 3. (a) is for the dotted curve and (b) is for the dashed curve

\begin{tabular}{rrrl}
\hline & $N_{s}\left(\mathrm{~cm}^{3}\right)$ & $Z_{s}$ & $r_{s}(\mathrm{~nm})$ \\
\hline 2500 & 1 & 0.18 \\
2210 & 1 & 1 \\
220 & 1 & 1.2 \\
36 & 1 & 1.4 \\
20 & 1 & 1.6 \\
10 & 1 & 1.8 \\
4 & 1 & 2 \\
& 1 & 0.18 \\
5000 & 1 & 1 \\
2210 & 1 & 1.2 \\
220 & 1 & 1.4 \\
36 & 1 & 1.6 \\
20 & 1 & 1.8 \\
10 & 1 & 2 \\
4 & -1 & 1 \\
2210 & -1 & 1.2 \\
220 & -1 & 1.4 \\
36 & -1 & 1.6 \\
20 & -1 & 1.8 \\
10 & -1 & 2 \\
4 & &
\end{tabular}

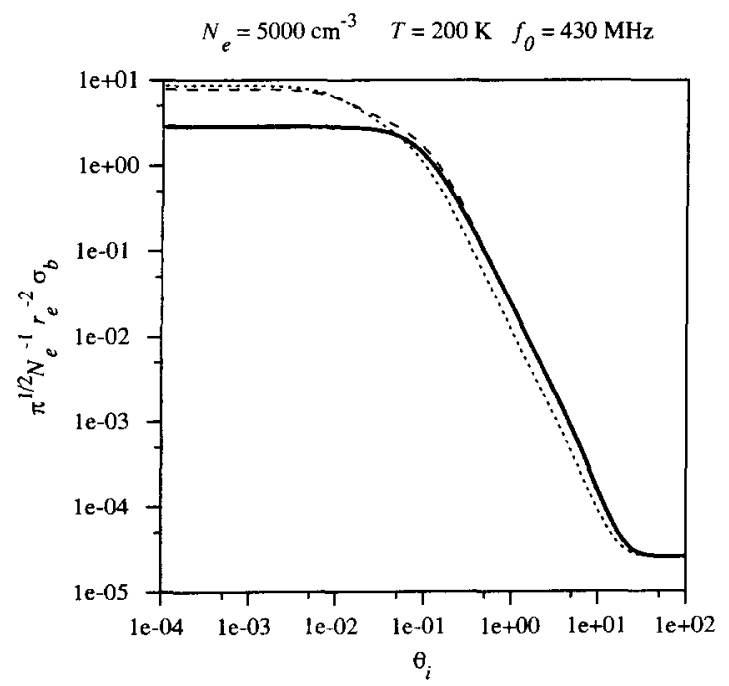

Fig. 3. Calculated incoherent scatter spectra for parameters given in Table 2. (a) is for the dotted curve and (b) is for the dashed curve. The thick line is the reference spectrum for Fig. 1.

three calculated spectra from Fig. 2 and add enough noise to them such that the signal-to-noise ratio (SNR) level is of the order of daytime D-region data taken with the Arecibo $430 \mathrm{MHz}$. ISR. We then attempt to fit a Lorentzian curve to each of them. The dust-free 


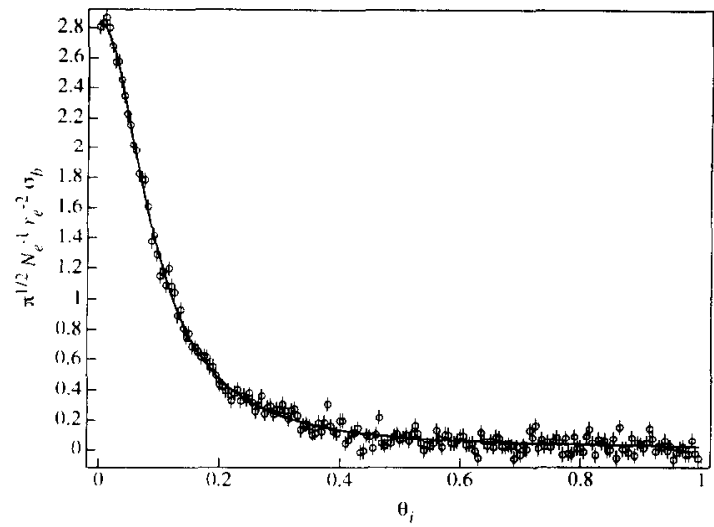

Fig. 4. Fit of a Lorentzian function (solid line) to the reference spectrum from the preceding three figures with noise (circles). The vertical bars in the circles denote the plus/minus standard deviations of the data points. Note that the axes are linear here.

model spectrum is strictly a Lorentzian, whereas the presence of dust forces the spectrum away from this function, so departures from an excellent fit would signal the presence of dust. The question is whether we would be able to recognize the difference between an "acceptable" fit and a failure of the dust-free model.

Figure 4 shows the Lorentzian fit to the simulated dust-free data spectrum (corresponding to the reference curve of the preceding figures plus noise). As expected the fit is excellent. It is also a reassuring indicating that eqn (19) does, indeed, reduce to the dust-free case. Figure 5 displays the fit to the simulated

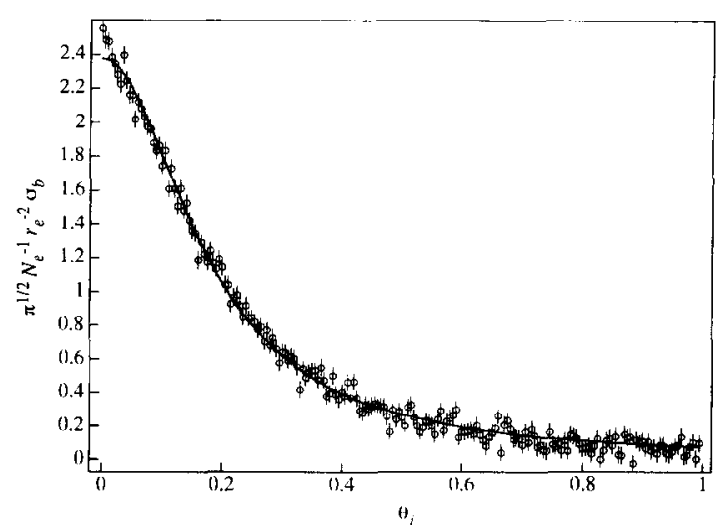

Fig. 5. The same as Fig. 4 except that the simulated data spectrum (circles) corresponds to the dotted curve from Fig. 2.

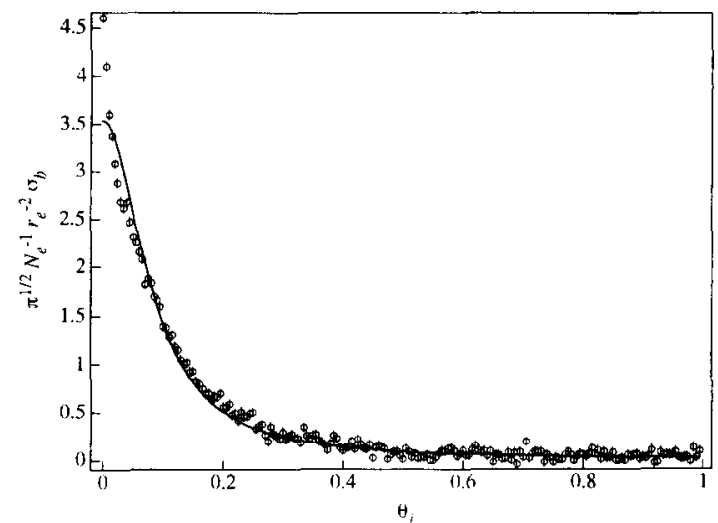

Fig. 6. The same as Fig. 4 except that the simulated data spectrum (circles) corresponds to the dashed curve from Fig. 2.

data spectrum corresponding to the dotted curve in Fig. 2. In this case the fit does not look so bad to the eye except at very low frequencies. Figure 6 shows the fit to the simulated data spectrum corresponding to the dashed curve in Fig. 2. Here the fit is clearly not good.

To quantify these results we produce a series of spectra for each of the above three cases with added random noise. The added random numbers have a standard deviation that approximates that obtained in the upper part of the D-region using the Arecibo $430 \mathrm{MHz}$ radar with about $20 \mathrm{~min}$ of integration under favorable conditions. They were generated using the routines ran2 and gasdev from Press et al. (1992). The numbers are independent from frequency to frequency since the radar data also have this property. We calculate for each case $Q$, which is the probability that the chi-square value for the fit is as poor as it is by chance. Thus values of $Q$ that average to 0.5 signify good fits, whereas low values indicate poor fits, i.e., that the model is wrong. Figure 7 displays the $Q$ values calculated for fits to each series of spectra. The reference spectra clearly fit the dust-free model well. The $Q$ values for the series generated from the spectrum corresponding to the dotted curve of Fig. 2 are significantly lower than those for the "perfect" fit, so under idcal conditions one would decide that these spectra do not fit the dust-free model well. However, under real experimental conditions one might be willing to accept $Q$ values as low as these, so this could be a borderline case for dust detection. However, a better test could be devised since the differences between the Lorentzian and the simulated data occur over a limited frequency range, and so it is likely that this case could be detected. 


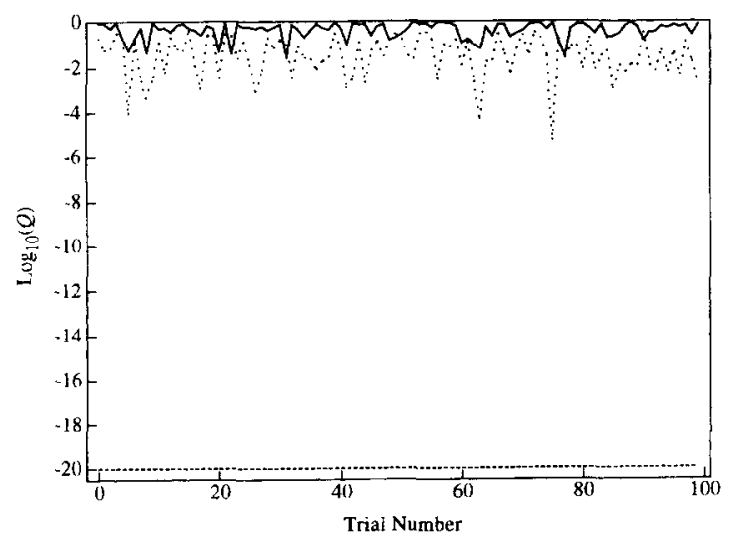

Fig. 7. The logarithm of $Q$ plotted for 100 fits of the Lorentzian function to simulated data spectra. The solid line corresponds to the reference curve (example fit given in Fig. 4), the dotted line corresponds to the example fit given in Fig. 5, and the dashed line corresponds to the example fit given in Fig. 6.

On the other hand, the $Q$ values of the series generated from the spectrum corresponding to the dashed curve of Fig. 2 are so close to zero that we had to add a small number to keep them on the log scale of Fig. 7. These spectra obviously do not fit the dust-free model and we should definitely be able to extract information about the deviation caused by the presence of dust.

\section{SUMMARY DISCUSSION}

It is clear from the spectral calculations that the main effect of charged dust on ISR spectra is to make them narrower. The exception is the case of very small negatively charged dust (or negative ions). Our simulations of real data and their fits to the dust-free Lorentzian spectrum provided good hope that with typical D-region SNR (at least for the Arecibo ISR) we should be able to extract information about meteoric dust. Of course, greater amounts of dust will produce clearer signatures, so we look forward to the predicted great Leonid meteor showers of 1998-99. With the world's most sensitive ISR, the Arecibo Observatory will be the ideal place to make those observations. The experiment will not be trivial, given the increasingly busy radio interference environment and the need to balance SNR with spectral resolution.

We would also like to comment on the systematic discrepancy between observed and calculated Dregion ISR spectral widths reported by Hansen et al. (1991). (A similar effect could be construed from mesospheric temperatures deduced from Arecibo ISR measurements (Zhou et al., 1996).) Since the spectral width is dependent on the diffusivities of the plasma species, which in turn are dependent on the temperature and collision frequency of the species, any systematic discrepancy must be due to one or more of the following: (1) The theory is wrong, (2) the temperatures used in the calculations are wrong, or (3) the collision frequency is wrong.

In response to the first point, ISR theory in the collisionless regime is well-tested and the agreement between theory and experiment is exceptionally good. How to include the effects of collisions into this theory has been a matter of debate over the years. Some have preferred to augment the kinetic theory with a relaxation term that conserves charged particles (Dougherty and Farley, 1963) or one that also conserves momentum (Waldteufel, 1965), while others have modeled the collisions with Brownian motion (Hagfors and Brockelman, 1971). The continuum approach (Tanenbaum, 1968) that we have followed is also valid in the collisional regime. Comparison of the theories have shown that they produce very similar results, although the spectral widths differ by slight amounts. If they are ordered with respect to increasing spectral width, then we have continuum, charge conservation, charge and momentum conservation, then Brownian (Fukuyama and Kofman, 1980; Ganguly and Coco, 1987). Since the most common theories used are the first two, we are already using the theories that produce the narrowest spectra. Therefore, even though we cannot assert with certainty that the theories in use are absolutely correct, we have no current alternative for a theory that produces narrower spectra.

As for the second point, if model temperatures are used in spectral calculations, a widening bias can be introduced if the model temperatures are higher than the actual temperatures. There is evidence to support this type of model bias for the summer mesopause, but not for other cases (Lübken and von Zahn, 1991). Furthermore, Hansen et al. (1991) used temperatures measured by lidar, so this argument cannot be used to discard their conclusion.

In examining the third point, we note that taking polarization as the sole interactive force between charged and neutral species is only strictly true at a temperature of absolute zero, even for small particles. An additional short-range quantum mechanical repulsion kicks in with increasing temperature. This effect has been traditionally ignored in the aeronomy community, because it was assumed that it was negligible below $300 \mathrm{~K}$. Although a change in the calculated ISR spectrum due to this effect will tend to 
narrow it, recent laboratory measurements of ion mobility suggest that it would be no greater than a few percent change (Viehland and Mason, 1995).

The point we would like to make here is that the presence of charged meteoric dust would introduce a narrowing bias to the observed ISR spectra (unless the dust radii are all smaller than about $1 \mathrm{~nm}$, in which case they would introduce a widening bias). For example, the dashed line spectrum of Fig. 2 has a $27 \%$ narrower width than the reference spectrum. (If one were trying to extract the temperature without taking into account the presence of dust, then one would arrive at a temperature value too low by $27 \%$.) And that curve was calculated from a fairly conservative dust size distribution. If much larger dust exist as suggested by the results of Kelley et al. (1997), then the resultant narrowing bias would be much stronger. In the special case of the summer polar mesopause, where ice particles as large as $100 \mathrm{~nm}$ can exist, ultranarrow ISR spectra have already been observed (Collis et al., 1988; Klostermeyer, 1994). However, note that they may be contaminated by other types of scattering mechanism, especially for the lower-frequency ISRs (Cho and Kelley, 1993).

We also note that the presence of charged dust could account for the difference between the ambipolar diffusion coefficients in meteor trains derived from VHF underdense echo decay and those calculated from lidar temperature measurements assuming the charged constituents to be only ions (Chilson et al., 1996). Since charged dust particles have lower diffusivities than ions, the lidar-derived diffusivities would be lowered to better match the values calculated from the echo decay times. Invoking the presence of ionized air molecules rather than metal ions will not account for the discrepancy, since the air molecules have lower mass and thus slightly higher mobilities. Chilson et al. (1996) quote a calculated value for the former that was higher than the measured ones for the latter. This is misleading because the equation used for calculation is only strictly valid at absolute zero temperature; at higher temperatures it overestimates the mobility. If calculated mobilities are compared with each other, or if measured values are compared with each other, then the metal ions should be less mobile than the air ions.

Acknowledgements - The Arecibo Observatory is part of the National Astronomy and Ionosphere Center, which is operated by Cornell University under a cooperative agreement with the National Science Foundation.

\section{REFERENCES}

Banks, P. M. and Kockarts, G. (1973) Aeronomy, Part A. Academic Press, New York, $430 \mathrm{pp}$.
Chilson, P. B., Czechowsky, P. and Schmidt, G. (1996) A comparison of ambipolar diffusion coefficients in meteor trains using VHF radar and UV lidar. Geophys. Res. Lett. 23, 2745-2748.

Cho, J. Y. N., Hall, T. M. and Kelley, M. C. (1992) On the role of charged aerosols in polar mesosphere summer echoes. J. Geophys. Res. 97, 875-886.

Cho, J. Y. N. and Kelley, M. C. (1993) Polar mesosphere summer radar echoes: observations and current theories. Rev. Geophys. 31, 243-265.

Cho, J. Y. N., Alcala, C. M., Kelley, M. C. and Swartz, W. E. (1996) Further effects of charged aerosols on summer mesospheric radar scatter. J. Atmos. Terr. Phys. 58, 661672.

Collis, P. N., Turunen, T. and Turunen, E. (1988) Evidence of heavy positive ions at the summer aretic mesopause from the EISCAT UHF incoherent scatter radar. Geophys. Res. Lett. 15, 148-151.

Dougherty, J. P. and Farley, D. T. (1960) A theory of incoherent scattering of radio waves by a plasma. Proc. Roy. Soc. London A259, 79--99.

Dougherty, J. P. and Farley, D. T. (1963) A theory of incoherent scattering of radio waves by a plasma, 3 , scattering in a partly ionized gas. J. Geophys. Res. 68, 5473-5486.

Fukuyama, K. and Kofman, W. (1980) Incoherent scattering of an electromagnetic wave in the mesosphere: a theoretical consideration. J. Geomagn. Geoelectr. 32, 67-81.

Ganguly, S. and Coco, D. (1987) Incoherent scattering from the collision dominated D-region. Comparison of theories with experimental data. J. Atmos. Terr. Phys. 49, 549563.

Hagfors, T. and Brockelman, R. A. (1971) A theory of collision dominated electron density fluctuations in a plasma with applications to incoherent scattering. Phys. Fhuids 14, 1143-1151.

Hagfors, T. (1992) Note on the scattering of electromagnetic waves from charged dust particles in a plasma. J. Atmos. Terr. Phys. 54, 333-338.

Hansen, G., Hoppe, U.-P., Turunen, E. and Pollari, P. (1991) Comparison of observed and calculated incoherent scatter spectra from the D region. Radio Sci. 26, 1153-1164.

Hunten, D. M., Turco, R. P. and Toon, O. B. (1980) Smoke and dust particles of meteoric origin in the mesosphere and stratosphere. J. Atmos. Sci. 37, 1342-1357.

Jensen, E. J. and Thomas, G. E. (1991) Charging of mesospheric particles: Implications for electron density and particle coagulation. J. Geophys. Res. 96, 18,603-18,615.

Kelley, M. C., Alcala, C. M. and Cho, J. Y. N. (1997) Detection of a meteor contrail and meteoric dust in the Earth's upper mesosphere. J. Atmos. Solar-Terr. Phys. 60 , 359-369.

Klostermeyer, J. (1994) The effect of ice particles on Thomson scattering from the polar summer mesopause region. Geophys. Res. Lett. 21, 2721-2724.

La Hoz, C. (1992) Radar scattering from dusty plasmas. Phys. Scr. 45, 529-534.

Love, S. G. and Brownlee, D. E. (1993) A direct measurement of the terrestrial mass accretion rate of cosmic dust. Science 262, 550-553.

Lübken, F.-J. and von Zahn, U. (1991) Thermal structure of the mesopause region at polar latitudes. J. Geophys. Res. 96, 20,841-20,857.

Mathews, J. D. (1978) ' The effect of negative ions on collisiondominated Thomson scattering. J. Geophys. 83, 505 512

Press, W. H., Teukolsky, S. A., Vetterling, W. T. and Flan- 
nery, B. P. (1992) Numerical Recipes in C: The Art of Scientific Computing. Cambridge University Press, New York, 994 pp.

Rosinski, J. and Snow, R. H. (1961) Secondary particulate matter from meteor vapors. J. Meteor. 18, 736-745.

Schulte, P. and Arnold, F. (1992) Detection of upper atmospheric negatively charged microclusters by a rocket-borne mass spcctrometer. Geophys. Res. Lett. 19, 2297-2300.

Schunk, R. W. (1975) Transport equations for aeronomy. Planet. Space Sci. 23, 437-485.

Solomon, S. (1990) Progress towards a quantitative understanding of Antarctic ozone depletion. Nature 347, 347354.

Tanenbaum, B. S. (1968) Continuum theory of Thomson scattering. Phys. Rev. 171, 215-221.

Thomas, G. E. (1991) Mesospheric clouds and the physics of the mesopause region. Rev. Geophys. 29, 553-575.

Trakhtengerts, V. Yu. and Demekhov, A. G. (1995) Nonequilibrium electron density fluctuations and wave scat- tering in the mesosphere. J. Atmos. Terr. Phys. 57, 11531164.

Turco, R. P., Toon, O. B., Hamill, P. and Whitten, R. C. (1981) Effects of meteoric debris on stratospheric aerosols. J. Geosphys. Res. 86, 1113-1128.

Viehland, L. A. and Mason, E. A. (1995) Transport properties of gaseous ions over a wide energy range, IV. At. Nucl. Data Tables 60, 37-95.

Waldteufel, P. (1965) Introduction de l'effect des collisions élastiques sur l'équation de Boltzmann d'un gaz faiblement ionizé-application á la diffusion incohérent d'une onde électromagnétique par l'ionosphére. Ann. Geophys. 21, $106-120$.

Zhou, Q. H., Sulzer, M. P., Tepley, C. A., Fesen, C. G., Roble, R. G. and Kelley, M. C. (1997) Neutral winds and temperature in the tropical mesosphere and lower thermosphere during January 1993 observation and comparison with TIME-GCM results. J. Geophys. Res. 102, 11,507-11,519. 